\title{
INVESTIGATION OF A SPATIAL DOUBLE PENDULUM: AN ENGINEERING APPROACH
}

\author{
S. BENDERSKY AND B. SANDLER
}

Received 24 May 2005; Accepted 4 July 2005

The behavior of a spatial double pendulum (SDP), comprising two pendulums that swing in different planes, was investigated. Movement equations (i.e., mathematical model) were derived for this SDP, and oscillations of the system were computed and compared with experimental results. Matlab computer programs were used for solving the nonlinear differential equations by the Runge-Kutta method. Fourier transformation was used to obtain the frequency spectra for analyses of the oscillations of the two pendulums. Solutions for free oscillations of the pendulums and graphic descriptions of changes in the frequency spectra were used for the dynamic investigation of the pendulums for different initial conditions of motion. The value of the friction constant was estimated experimentally and incorporated into the equations of motion of the pendulums. This step facilitated the comparison between the computed and measured oscillations.

Copyright (C) 2006 S. Bendersky and B. Sandler. This is an open access article distributed under the Creative Commons Attribution License, which permits unrestricted use, distribution, and reproduction in any medium, provided the original work is properly cited.

\section{Introduction}

Research on different kinds of pendulum, including the double pendulum in a plane (PDP), has spanned more than three centuries, starting with the invention of the pendulum clock in 1657 . Two hundred a fifty years later, research on a pendulum with vertical oscillations of the hinge showed the influence of the moving suspension point on the oscillations [15]. These early studies were followed by investigations of other types of pendulum, such as, an inverted pendulum with an oscillating suspension point under various conditions $[4,10]$; the PDP $[7,3]$, including the so-called spherical pendulum [5]; and various pendulums with an oscillating motion of the base hinge $[13,14]$. In addition, theoretical studies were conducted on nonlinear motions of the elastic pendulum or the swinging spring (motions of a three-dimensional pendulum) $[2,6,11]$. Lynch [6], the author of one of these theoretical studies, wrote "The equations of motion are easy to write down but, in general, impossible to solve analytically. For finite amplitudes, the

Hindawi Publishing Corporation

Discrete Dynamics in Nature and Society

Volume 2006, Article ID 25193, Pages 1-22

DOI 10.1155/DDNS/2006/25193 
motion of the system exhibits chaos, and predictability is severely limited." The same may be said of the spatial double pendulum (SDP) system.

In the current study, computer-aided analyses were applied to the study of the SDP. This system is unusual in that both pendulums execute planar motions, but the planes of these motions are distinct. The SDP is thus a simple mechanical system with complex dynamics. It comprises two masses suspended at two fixed points by mass-less rigid rods that move freely under gravity.

It is not difficult to express the equations of motion, but it is impossible to solve them analytically. For nonlinear cases, we have shown that a combined numerical-analytical approach enables us to describe some features of the complex motion of the two pendulums under consideration and thereby to obtain estimations of some important system characteristics (number of frequencies and their values). The approach developed in this work enables us

(a) to obtain approximate estimations of the system's behavior, since there are still no strict mathematical means at the designer's disposal for solving the mathematical equations; and

(b) to find the desired domain of action of the system, and in this narrow domain to use more powerful and exact techniques, if such methods do indeed exist.

Movement equations (mathematical model) were defined for the SDP, and, on the assumption of a linear system, an analytical study of the vibrations of the system was conducted. The results obtained were compared with experimental results. For the linear case, the vibrations of the SDP were described both analytically and graphically.

For the non-linear oscillations mode, Fourier transformation was applied for the examination of the frequency spectra of the two pendulums comprising the double pendulum. Graphical solutions for free oscillations and graphical descriptions of the change in frequency constituted the basis of the research on the dynamics of the SDP for different parameter values and initial conditions. In addition, the value of the friction constant for the experimental system was estimated and used in the analytical description of the behavior of the two pendulums of the SDP.

The computation layout for the SDP, that is, for a constrained system of two particles is shown in Figure 1.1(a). The hinge of the first pendulum swinging in the $X-Y$ plane is located at point $O$. This pendulum comprises a mass-less rigid $\operatorname{rod} L_{1}$ and a point mass $m_{1}$. At point $O^{\prime}$ (which coincides with the mass center of the mass $m_{1}$ ), a second hinge is mounted in such a way that the coordinate system $X^{\prime} Y^{\prime} Z^{\prime}$ can rotate around the $Y^{\prime \prime}$ axis. The angle between the oscillation planes is designated $\Psi$. The second hinge serves as the suspension point for the second pendulum, consisting of a $\operatorname{rod} L_{2}$ and a mass $m_{2}$. For $\Psi=0$, this model becomes a PDP and $X^{\prime} Y^{\prime} Z^{\prime}$ coincides with $X^{\prime \prime} Y^{\prime \prime} Z^{\prime \prime}$. A photograph of the experimental device corresponding to the model described above is presented in Figure 1.1(b).

The central question that we set out to answer in this study is: What is the influence of the angle $\Psi$ on the behavior of the SDP? To find a solution to this question, the following steps were taken: formulating the dynamic model by applying the Lagrange method and finding solutions for small and non-small values of the angles $\varphi_{i}(i=1,2)$, respectively; obtaining graphical solutions for free oscillations and graphical descriptions of the 


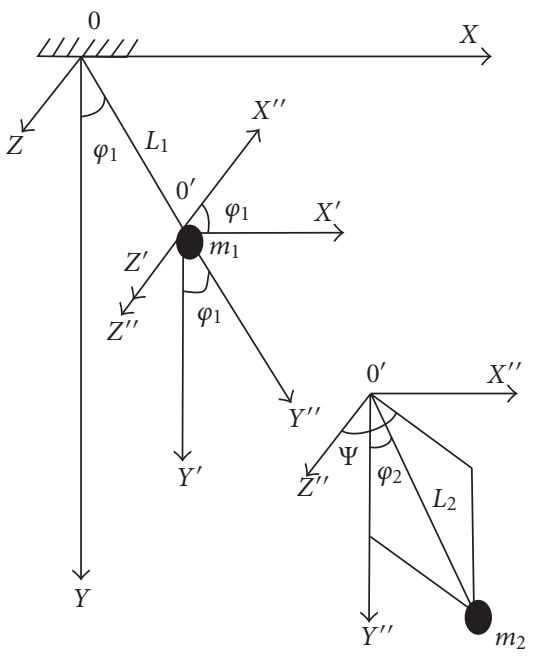

(a)

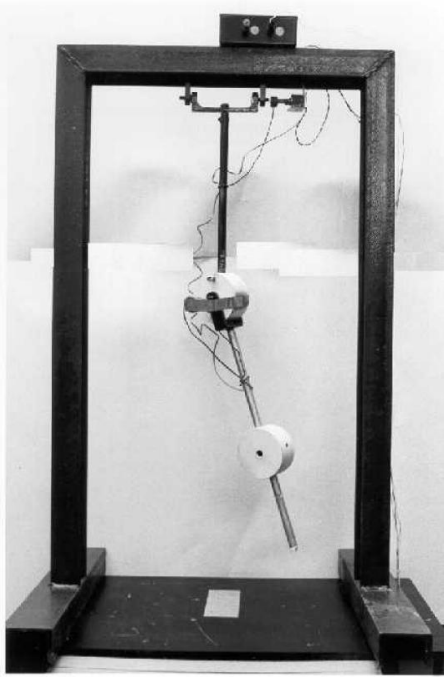

(b)

Figure 1.1. (a) Model and computation layout of an SDP; (b) photograph of the SDP.

changes in the frequency spectra by using Fourier transformations for different parameter values and initial conditions.

\section{Mathematical model}

2.1. General model. The following equations for free oscillations without damping (obtained by the Lagrange method) govern the behavior of the SDP:

$$
\frac{d}{d t}\left(\frac{\partial T}{\partial \dot{q}_{i}}\right)-\frac{\partial T}{\partial q_{i}}+\frac{\partial P}{\partial q_{i}}=Q_{i}, \quad i=1,2
$$

where $T$ and $P$ are the kinetic and potential energy, respectively, as given by

$$
\begin{aligned}
T= & \frac{1}{2}\left(m_{1}+m_{2}\right) L_{1}^{2} \dot{\varphi}_{1}^{2}+\frac{1}{2} m_{2} L_{2}^{2} \dot{\varphi}_{2}^{2}+\frac{1}{2} m_{2} L_{2}^{2} \dot{\varphi}_{1}^{2} \cos ^{2} \Psi \sin ^{2} \varphi_{2} \\
& +\frac{1}{2} m_{2} L_{2}^{2} \dot{\varphi}_{1}^{2} \cos ^{2} \varphi_{2}+m_{2} L_{2}^{2} \dot{\varphi}_{1} \dot{\varphi}_{2} \cos \psi+m_{2} L_{1} L_{2} \dot{\varphi}_{1} \dot{\varphi}_{2} \cos \Psi \cos \varphi_{2}+m_{2} L_{1} L_{2} \dot{\varphi}_{1}^{2} \cos \varphi_{2}, \\
P= & m_{1} g L_{1}\left(1-\cos \varphi_{1}\right)+m_{2} g L_{1}\left(1-\cos \varphi_{1}\right) \\
& +m_{2} g L_{2}\left(1-\cos \varphi_{1} \cos \varphi_{2}\right)+m_{2} g L_{2} \cos \Psi \sin \varphi_{1} \sin \varphi_{2},
\end{aligned}
$$

where $\varphi_{1}$ and $\varphi_{2}$ are the deviation angles of pendulums 1 and 2, respectively, and $g$ is acceleration due to gravity. For deriving the movement equations for our SDP system, we 
4 Investigation of a spatial double pendulum

defined

$$
\begin{gathered}
\Psi=\text { const } \quad Q_{i}=0 ; \\
q_{1}=\varphi_{1}(t) ; \quad q_{2}=\varphi_{2}(t) ; \quad \dot{q}_{1}=\dot{\varphi}_{1}(t) ; \quad \dot{q}_{2}=\dot{\varphi}_{2}(t) .
\end{gathered}
$$

The equations governing motion may then be expressed as follows:

$$
\begin{aligned}
& \ddot{\varphi}_{1}[\left.\left(m_{1}+m_{2}\right) L_{1}^{2}+m_{2} L_{2}^{2} \cos ^{2} \Psi \sin ^{2} \varphi_{2}+m_{2} L_{2}^{2} \cos ^{2} \varphi_{2}+2 m_{2} L_{1} L_{2} \cos \varphi_{2}\right] \\
&+\ddot{\varphi}_{2}\left[m_{2} L_{2}^{2} \cos \Psi+m_{2} L_{1} L_{2} \cos \Psi \cos \varphi_{2}\right] \\
&+\dot{\varphi}_{1} \dot{\varphi}_{2}\left[2 m_{2} L_{2}^{2} \cos ^{2} \Psi \cos \varphi_{2} \sin \varphi_{2}-2 m_{2} L_{2}^{2} \cos \varphi_{2} \sin \varphi_{2}-2 m_{2} L_{1} L_{2} \sin \varphi_{2}\right] \\
&-\dot{\varphi}_{2}^{2} m_{2} L_{1} L_{2} \cos \Psi \sin \varphi_{2} \\
&+\left(m_{1}+m_{2}\right) g L_{1} \sin \varphi_{1}+m_{2} g L_{2}\left(\cos \Psi \cos \varphi_{1} \sin \varphi_{2}+\sin \varphi_{1} \cos \varphi_{2}\right)=0, \\
& \ddot{\varphi}_{2} m_{2} L_{2}^{2}+\ddot{\varphi}_{1}\left[m_{2} L_{2}^{2} \cos \Psi+m_{2} L_{1} L_{2} \cos \Psi \cos \varphi_{2}\right] \\
& \quad+\dot{\varphi}_{1}^{2}\left[m_{2} L_{2}^{2} \cos \varphi_{2} \sin \varphi_{2}^{-} m_{2} L_{2}^{2} \cos { }^{2} \Psi \sin \varphi_{2} \cos \varphi_{2}+m_{2} L_{1} L_{2} \sin \varphi_{2}\right] \\
& \quad+m_{2} g L_{2}\left(\cos \Psi \sin \varphi_{1} \cos \varphi_{2}+\cos \varphi_{1} \sin \varphi_{2}\right)=0 .
\end{aligned}
$$

There are a number of problems associated with the numerical and analytical solutions to this system of nonlinear equations (2.4). Iterative techniques are traditionally used to obtain numerical solutions, but nearly all iterative methods are sensitive to the initial solutions. Solutions to the linear model of SDP and to the pure nonlinear case are discussed below. The perturbation expansion method for small parameters-widely used to analyze simple nonlinear problems $[8,9]$-is not really effective for our mechanism.

2.2. Linear model. We therefore set out to solve our mathematical model for an SPD analytically for small angles and to obtain the natural frequencies and oscillation modes. Assuming

$$
\sin \varphi \approx \varphi, \quad \cos \varphi \approx 1, \quad \dot{\varphi}^{2} \approx 0,
$$

we may write

$$
\begin{aligned}
& \ddot{\varphi}_{1}\left[\left(m_{1}+m_{2}\right) L_{1}^{2}+m_{2} L_{2}^{2}+2 m_{2} L_{1} L_{2}\right]+\ddot{\varphi}_{2}\left[m_{2} L_{2}^{2} \cos \Psi+m_{2} L_{1} L_{2} \cos \Psi\right] \\
& \quad+\varphi_{1}\left(m_{1}+m_{2}\right) g L_{1}+\varphi_{2} m_{2} g L_{2} \cos \Psi+\varphi_{1} m_{2} g L_{2}=0, \\
& \ddot{\varphi}_{2} m_{2} L_{2}^{2}+\ddot{\varphi}_{1}\left[m_{2} L_{2}^{2} \cos \Psi+m_{2} L_{1} L_{2} \cos \Psi\right]+\varphi_{1} m_{2} g L_{2} \cos \Psi+\varphi_{2} m_{2} g L_{2}=0 .
\end{aligned}
$$


In matrix form, when $[\ddot{\varphi}]$ and $[\varphi]$ are column vectors and $[M]$ and $[k]$ are square matrixes, expression (2.6) may be rewritten as

$$
\begin{gathered}
{[M] \cdot[\ddot{\varphi}]+[k] \cdot[\varphi]=0} \\
M_{11}=\left(m_{1}+m_{2}\right) L_{1}^{2}+m_{2} L_{2}^{2}+2 m_{2} L_{1} L_{2} ; \quad M_{12}=m_{2} L_{2}^{2} \cos \Psi+m_{2} L_{1} L_{2} \cos \Psi ; \\
M_{21}=m_{2} L_{2}^{2} \cos \Psi+m_{2} L_{1} L_{2} \cos \Psi ; \quad M_{22}=m_{2} L_{2}^{2} ; \\
k_{11}=\left(m_{1}+m_{2}\right) g L_{1}+m_{2} g L_{2} ; \quad k_{12}=m_{2} g L_{2} \cos \Psi ; \\
k_{21}=m_{2} g L_{2} \cos \Psi ; \quad k_{22}=m_{2} g L_{2} .
\end{gathered}
$$

The transformations for amplitudes $A_{1}$ and $A_{2}$, and frequencies $\omega_{1}$ and $\omega_{2}$, - given in (2.8) to (2.12) —made below show the following possible solution:

$$
\begin{aligned}
& \varphi_{1}=A_{1} \sin (\omega \cdot t+\gamma) ; \quad A_{1} \neq A_{2}=\mathrm{const} \\
& \varphi_{2}=A_{2} \sin (\omega \cdot t+\gamma), \\
& \left(k_{11}-M_{11} \omega^{2}\right) A_{1}+\left(k_{12}-M_{12} \omega^{2}\right) A_{2}=0 ; \\
& \left(k_{21}-M_{21} \omega^{2}\right) A_{1}+\left(k_{22}-M_{22} \omega^{2}\right) A_{2}=0 .
\end{aligned}
$$

From (2.8), we find the expressions for the natural frequencies:

$$
\begin{gathered}
\omega^{4}\left[M_{11} M_{22}-M_{12}^{2}\right]+\omega^{2}\left[2 k_{12} M_{12}-k_{11} M_{22}-k_{22} M_{11}\right]+\left[k_{11} k_{22}-k_{12}^{2}\right]=0 ; \\
D=\left(L_{2}+L_{1}\right)^{2}\left[m_{2} g L_{1} L_{2}\left(m_{1}+m_{2}\right)+2 m_{2}^{2} g L_{2}^{2} \sin ^{2} \Psi\right]^{2} \\
-4\left[m_{2} L_{1}^{2} L_{2}^{2}\left(m_{1}+m_{2} \sin ^{2} \Psi\right)\right. \\
\left.\quad+m_{2}^{2} L_{2}^{3} \sin ^{2} \Psi\left(L_{2}+2 L_{1}\right)\right]\left[m_{2}^{2} g^{2} L_{2}^{2} \sin ^{2} \Psi+m_{2} g^{2} L_{1} L_{2}\left(m_{1}+m_{2}\right)\right] \\
\quad \\
=\sqrt{\frac{\left(L_{2}+L_{1}\right)\left[m_{2} g L_{1} L_{2}\left(m_{1}+m_{2}\right)+2 m_{2}^{2} g L_{2}^{2} \sin ^{2} \Psi\right] \pm \sqrt{D}}{2 m_{2} L_{1}^{2} L_{2}^{2}\left(m_{1}+m_{2} \sin ^{2} \Psi\right)+2 m_{2}^{2} L_{2}^{3} \sin ^{2} \Psi\left(L_{2}+2 L_{1}\right)}}
\end{gathered}
$$

For example, for the parameters $\Psi=30^{\circ}, L_{1}=L_{2}=0.3 \mathrm{~m}, m_{1}=m_{2}=1.57 \mathrm{~kg}$, the natural frequencies become

$$
\begin{aligned}
& \underline{\underline{\omega_{1}=7.91\left[\mathrm{~s}^{-1}\right]}} \\
& \underline{\underline{\omega_{2}=4.387\left[\mathrm{~s}^{-1}\right]}}
\end{aligned} \Longrightarrow \begin{gathered}
T_{1}=0.794[\mathrm{~s}] \\
T_{2}=1.4315[\mathrm{~s}]
\end{gathered} \Longrightarrow \underline{\underline{\underline{f_{1}=1.26[\mathrm{~Hz}]}}}
$$


where $\omega_{1}$ and $\omega_{2}$ are frequencies in radians per second; $f_{1}$ and $f_{2}$ are frequencies in oscillations per second; and $T_{1}$ and $T_{2}$ are periods of oscillations in seconds.

The solution of (2.8) gives two combined analytical solutions. Let us suppose an "exact" analytical solution in the form

$$
\begin{aligned}
& \varphi_{1}=A_{11} \sin \left(\omega_{1} \cdot t+\gamma_{1}\right)+A_{12} \sin \left(\omega_{2} \cdot t+\gamma_{2}\right), \\
& \varphi_{2}=A_{21} \sin \left(\omega_{1} \cdot t+\gamma_{1}\right)+A_{22} \sin \left(\omega_{2} \cdot t+\gamma_{2}\right),
\end{aligned}
$$

where from (2.8)-(2.11):

$$
\begin{aligned}
& \left(k_{11}-M_{11} \omega_{1}^{2}\right) A_{11}+\left(k_{12}-M_{12} \omega_{2}^{2}\right) A_{12}=0 ; \\
& \left(k_{21}-M_{21} \omega_{1}^{2}\right) A_{21}+\left(k_{22}-M_{22} \omega_{2}^{2}\right) A_{22}=0 .
\end{aligned}
$$

Then, the ratios of the amplitudes as a function of $\Psi s$ derived from expression (2.12) are

$$
\begin{gathered}
\frac{A_{11}}{A_{12}}=\frac{m_{2} L_{2}\left[\omega_{2}^{2}\left(L_{2}+L_{1}\right)-g\right]}{\left(m_{1}+m_{2}\right) L_{1}\left(g-\omega_{1}^{2} L_{1}\right)+m_{2} L_{2}\left[g-\omega_{1}^{2}\left(L_{2}+2 L_{1}\right)\right]} \cos \Psi \\
\frac{A_{22}}{A_{21}}=\frac{g-\omega_{1}^{2}\left(L_{2}+L_{1}\right)}{\omega_{2}^{2} L_{2}-g} \cos \Psi .
\end{gathered}
$$

Equations (2.9) and (2.13) are suitable for all masses and lengths of the pendulums for small oscillation angles of mass deviations, but not for all $\Psi$ values. From expression (2.13), when $\Psi \approx \pi / 2$ and $\cos \Psi \rightarrow 0$, the analytical description amplitude ratio of the SDP becomes unreal. Thus, mathematically, there is no possibility of moving the second pendulum, while the physical possibility obviously exists. In this extreme case, classical linearization does not work. We seek the explanations for this point, and find it in nonlinearities of problem for this case.

The graphical solution for the general model of the movement of pendulums is shown in Figure 2.1 [See (2.4) for specific, previously described parameters and initial conditions.] As was mentioned earlier, (2.9) and (2.10), the two values of the frequencies are identical for both pendulums, but with different energy. For example, the exact analytical solution for the SDP for the parameters shown previously when initial angular velocities are zero and free oscillations are considered for small angles such as $\varphi_{1}=2^{\circ}$ and $\varphi_{2}=3^{\circ}$, is given in the form of (2.14):

$$
\left\{\begin{array}{l}
\varphi_{1} \\
\varphi_{2}
\end{array}\right\}=\left[\begin{array}{cc}
1 & 1 \\
-2.68 & 0.37
\end{array}\right]\left\{\begin{array}{c}
-0.0128 \sin (7.91 t+\pi / 2) \\
0.0478 \sin (4.387 t+\pi / 2)
\end{array}\right\} .
$$

\section{Application of numeralytics solutions}

The general nonlinear and linear forms of a mathematical model (movement equations) for the SDP were shown in the previous section. For the linear case, we accepted the conventional analytical expression for the natural frequencies and limitations of the amplitude ratios influencing the angle $\Psi$. For the nonlinear case, we took a numeralytical approach [12] by applying the Matlab programs package. 

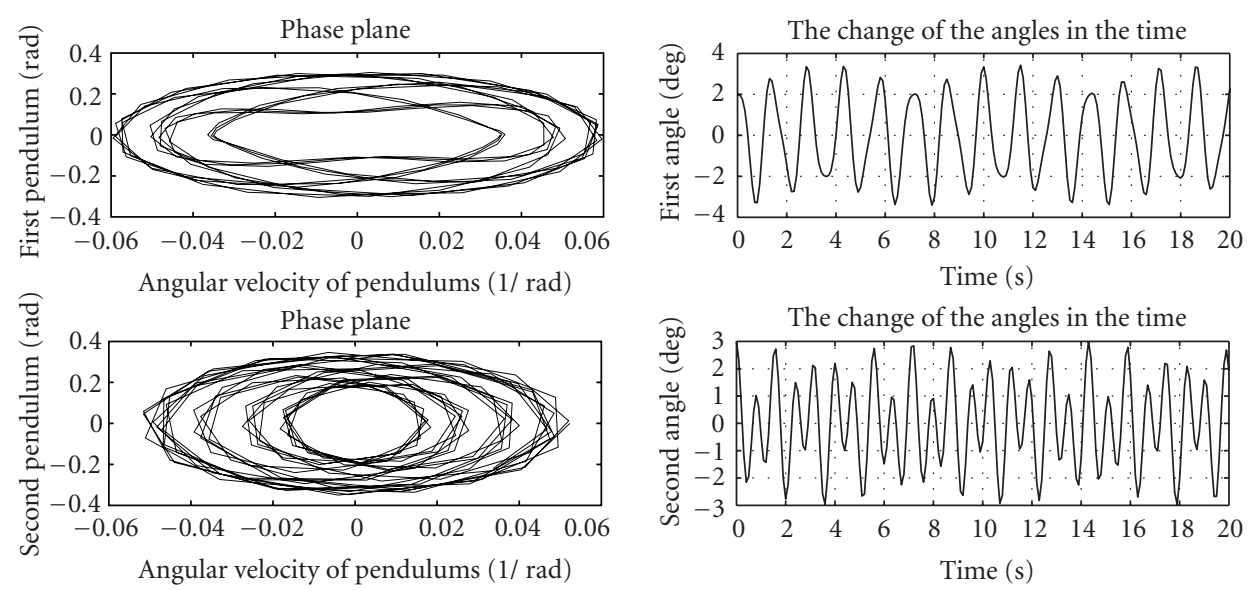

(a)

(b)
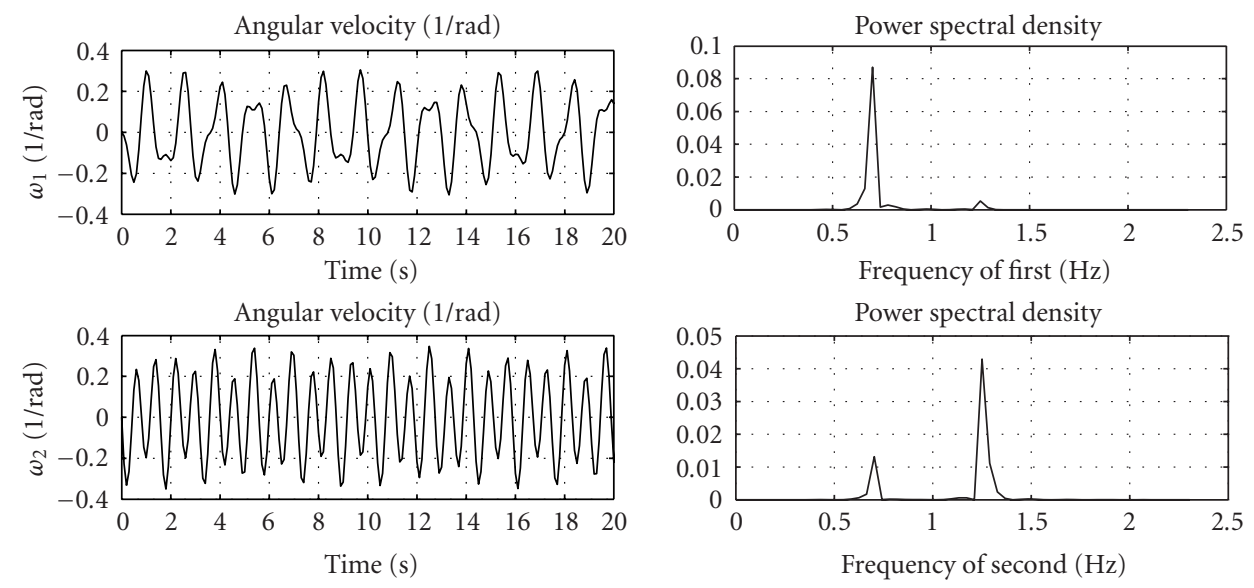

(c)

(d)

Figure 2.1. (a) "Phase portraits" for the two pendulums; (b) swinging angles $\varphi_{1}(t)$ and $\varphi_{2}(t)$; (c) swinging speeds $\dot{\varphi}_{1}(t)$ and $\dot{\varphi}_{2}(t)$; (d) spectrum distribution for the two pendulums.

Since it is not possible to answer the central question-the influence of the angle $\Psi$ on the behavior of the SDP-directly from the given equation system (2.4), we use the Runge-Kutta method for solving the nonlinear equations and Fourier transformation for investigation of the frequency spectra of the two pendulums. The solutions are represented graphically for a wide range of initial conditions, values of the parameters, and different values of the angle $\Psi$. Here, we show solutions for $\Psi=0^{\circ}, \Psi=15^{\circ}, \Psi=30^{\circ}, \Psi=$ $60^{\circ}$ and $\Psi=90^{\circ}$. Graphical solutions for the general model of the movement equation (2.4), for the described parameters, and for different initial conditions are shown in Figures 3.1-3.5. 

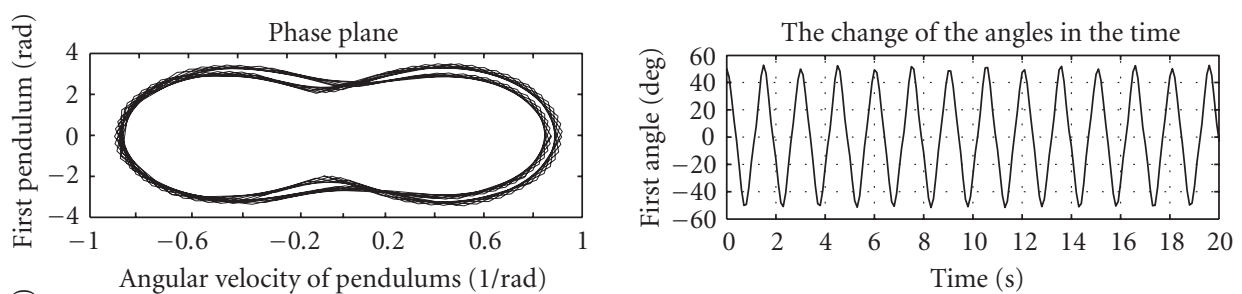

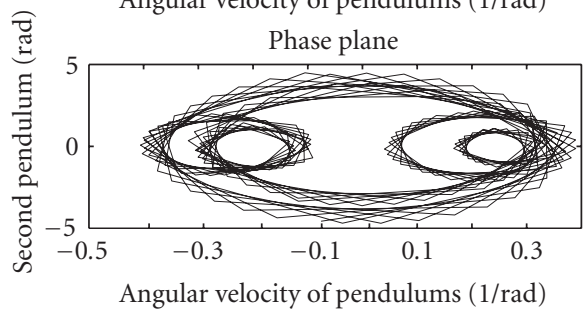

(a)

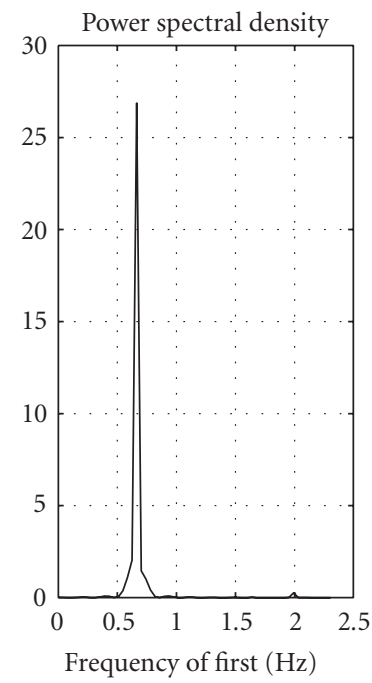

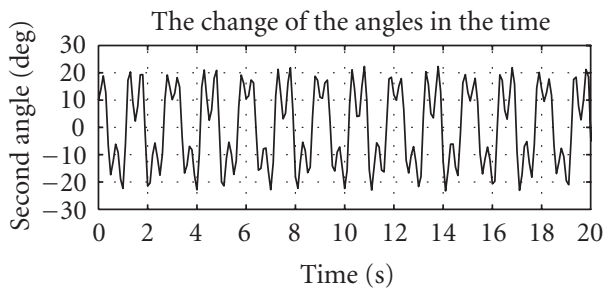

(b)

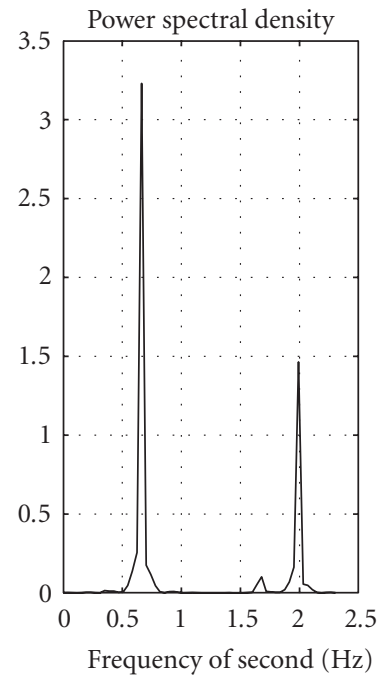

(c)

Figure 3.1. (a) "Phase portraits" for the two pendulums; (b) swinging angles $\varphi_{1}(t)$ and $\varphi_{2}(t)$; (c) oscillation spectrum distributions for the two pendulums. Parameters are $m_{1}=m_{2}=1.57 \mathrm{~kg}, L_{1}=$ $L_{2}=0.3 \mathrm{~m}, \Psi=0^{\circ}$. Initial conditions are $\varphi_{1}=50^{\circ}, \varphi_{2}=0^{\circ}$.

Let us now consider the case shown in Figures 3.3 and 3.3(c) in which the behavior of the first and second pendulums is governed by at least three and four frequencies, respectively. In this case, the "phase portraits," especially that of the second pendulum, are far from to be elliptic, that is, the oscillations are close to non-linear. An examination of Figures 3.1 and 3.2 shows that the number of peaks is not equal for the two pendulums. 

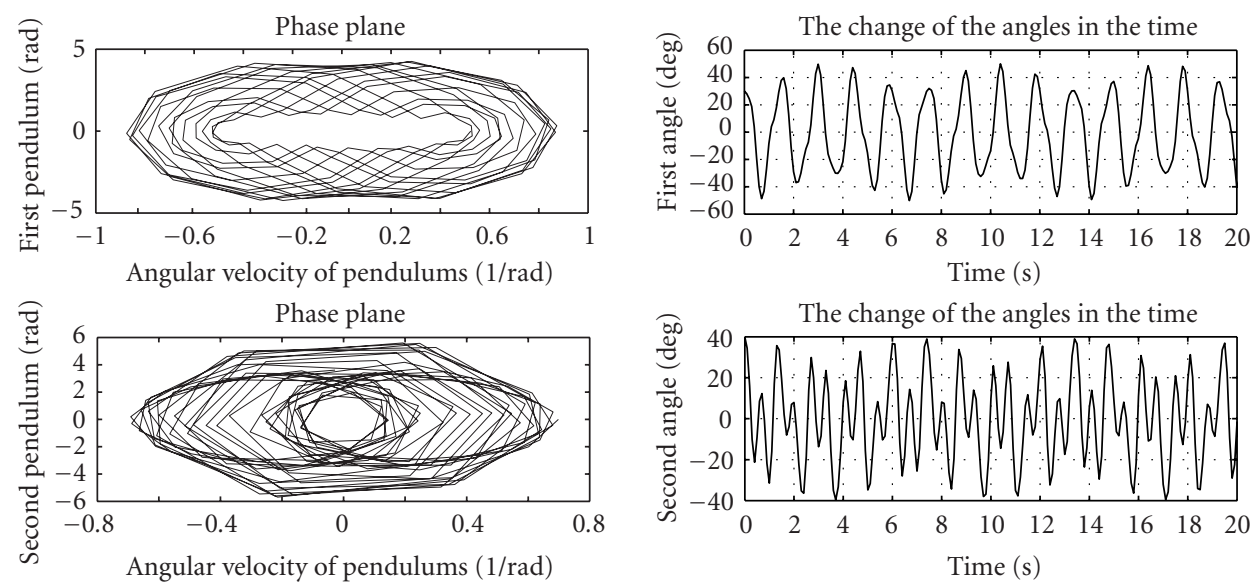

(a)

(b)
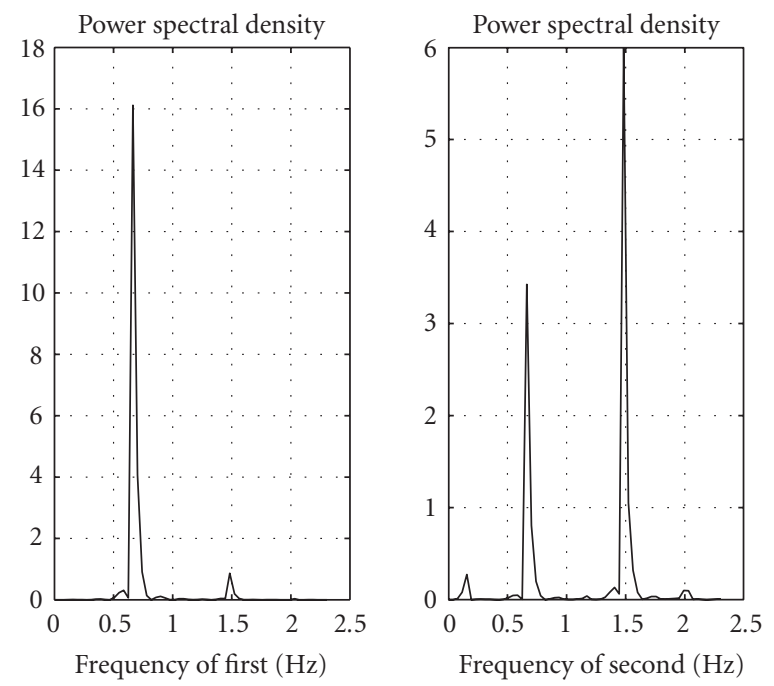

(c)

Figure 3.2. (a) "Phase portraits" for the two pendulums; (b) swinging angles $\varphi_{1}(t)$ and $\varphi_{2}(t)$; (c) oscillation spectrum distributions for the two pendulums. Parameters are $m_{1}=m_{2}=1.57 \mathrm{~kg}, L_{1}=$ $L_{2}=0.3 \mathrm{~m}, \Psi=15^{\circ}$. Initial conditions are $\varphi_{1}=30^{\circ}, \varphi_{2}=40^{\circ}$.

From an analysis of Figures 3.5(a) and 3.5(c), we may conclude that (1) the phase portraits of pendulums 1 and 2 fit linear oscillations (the "phase portraits" are almost elliptical) and (2) both pendulums have one markedly exposed frequency peak. Figures 3.4(a) and 3.4(c) illustrate the case in which the "phase portrait" of the first pendulum reflects linear oscillations, as in Figure 3.5(a), whereas the second pendulum is governed by two 

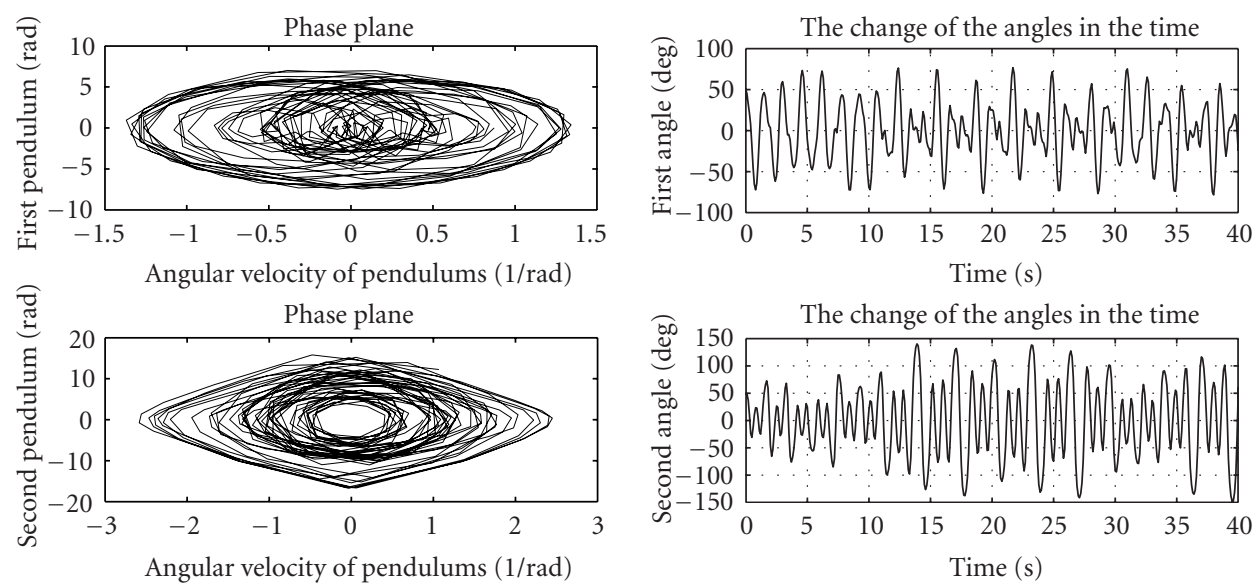

(a)

(b)
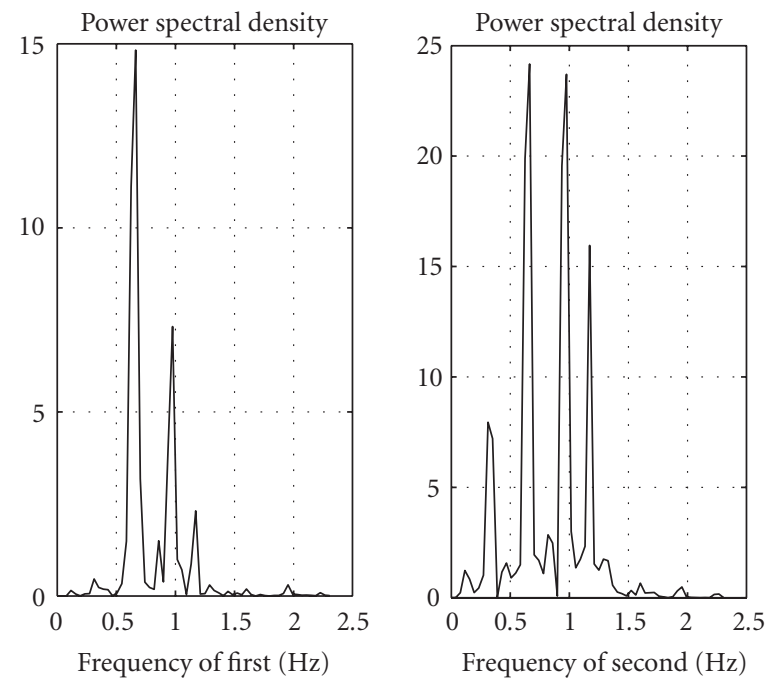

(c)

Figure 3.3. (a) "Phase portraits" for the two pendulums; (b) swinging angles $\varphi_{1}(t)$ and $\varphi_{2}(t)$; (c) oscillation spectrum distributions for the two pendulums. Parameters are $m_{1}=m_{2}=1.57 \mathrm{~kg}, L_{1}=$ $L_{2}=0.3 \mathrm{~m}, \Psi=30^{\circ}$. Initial conditions are $\varphi_{1}=50^{\circ}, \varphi_{2}=50^{\circ}$.

frequencies. Even so, the oscillations of both pendulums remain close to linear as a result of the harmonic form of the oscillations of either the first or the second pendulum. As opposed to solutions for the linear case, the nonlinear case is characterized by serendipitous numbers and values of the frequencies for both pendulums of the system. In such a situation, the engineering numeralytical approach, based on statistical investigation of 

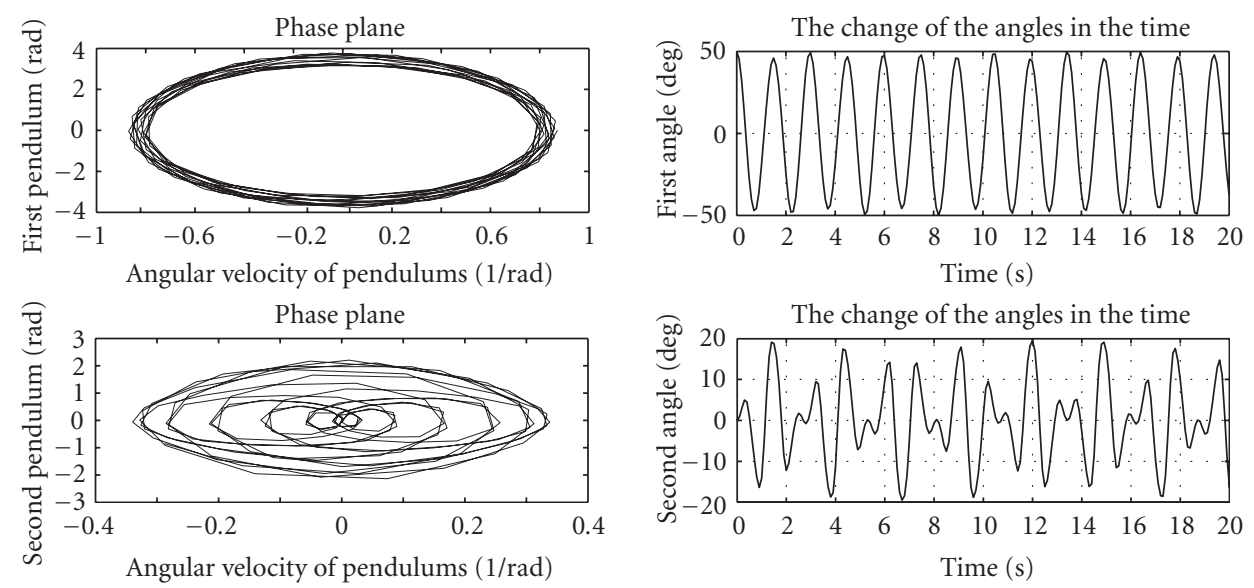

(a)

(b)
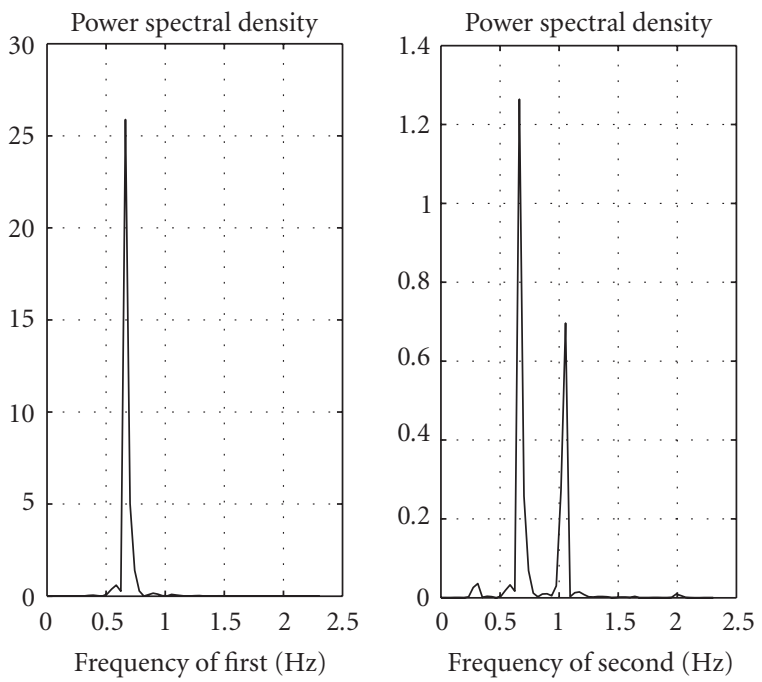

(c)

Figure 3.4. (a) "Phase portraits" for the two pendulums; (b) swinging angles $\varphi_{1}(t)$ and $\varphi_{2}(t)$; (c) oscillation spectrum distributions for the two pendulums. Parameters are $m_{1}=m_{2}=1.57 \mathrm{~kg}, L_{1}=$ $L_{2}=0.3 \mathrm{~m}, \Psi=60^{\circ}$. Initial conditions are $\varphi_{1}=50^{\circ}, \varphi_{2}=0^{\circ}$.

numerical (or/and graphical) solution data, may be helpful. Randomized approaches for investigating the behavior of pendulums have previously been reported [1]. In our case, the study of the behavior of the SDP, especially the number of frequencies for the two pendulums versus the angle between the oscillation planes of pendulums, is an excellent example of a case in which the elements of random function theory may be applied. 

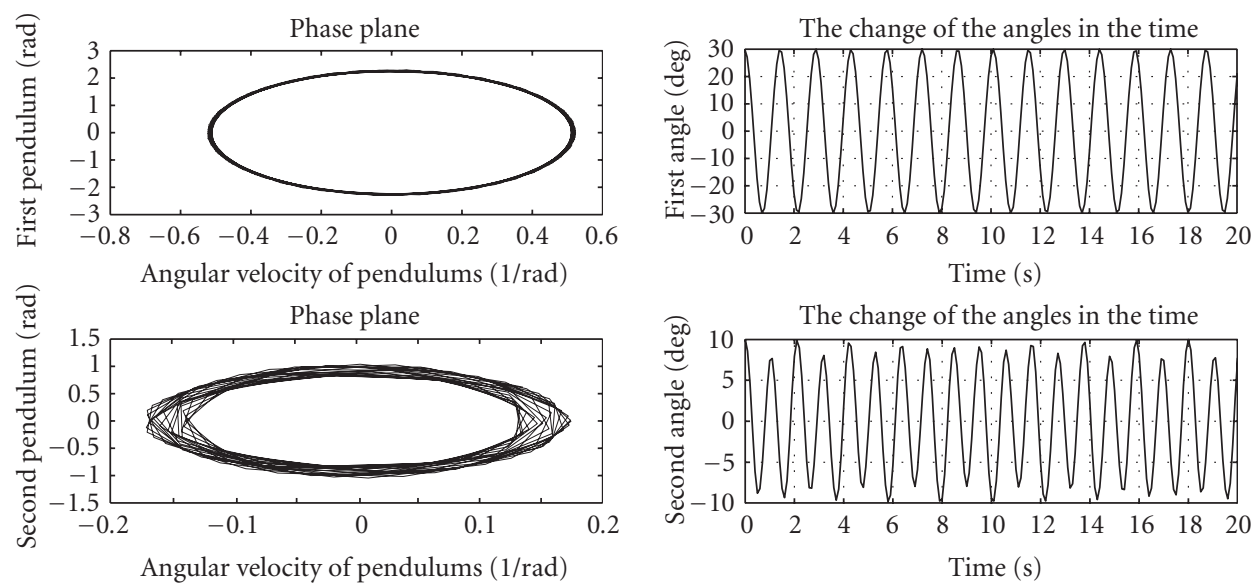

(a)

(b)
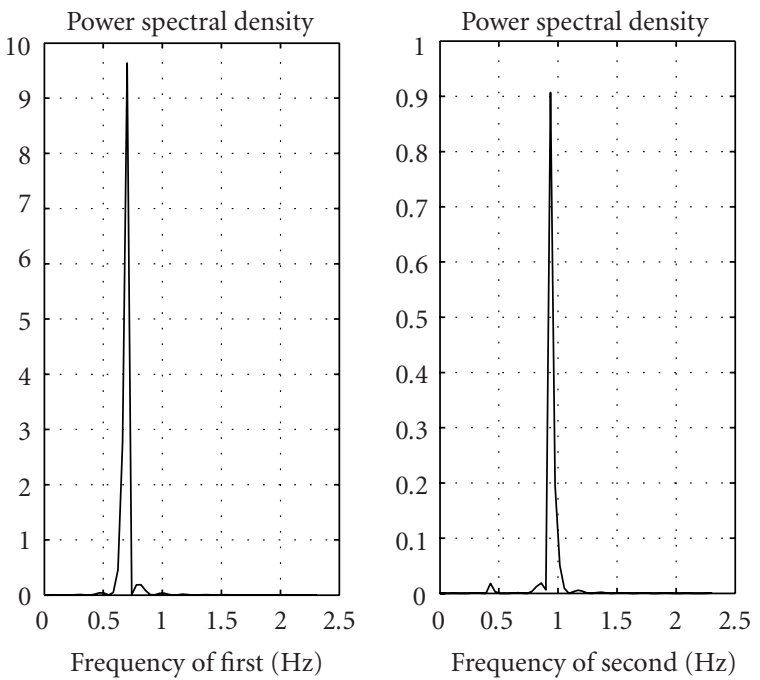

(c)

Figure 3.5. (a) "Phase portraits" for the two pendulums; (b) swinging angles $\varphi_{1}(t)$ and $\varphi_{2}(t)$; (c) oscillation spectrum distributions for the two pendulums. Parameters are $m_{1}=m_{2}=1.57 \mathrm{~kg}, L_{1}=$ $L_{2}=0.3 \mathrm{~m}, \Psi=90^{\circ}$. Initial conditions are $\varphi_{1}=30^{\circ}, \varphi_{2}=10^{\circ}$.

\section{Behavior of the SDP}

The "phase portraits" of the computed motion of the SDP given in Figures 3.1-3.5 illustrate the differences in the frequencies of oscillations of the pendulums with changes in the angle $\Psi$. It follows from the diagrams that for $\Psi=90^{\circ}$ both pendulums oscillate very closely to the harmonic mode (even when the initial deviations $\varphi_{1}$ and $\varphi_{2}$ are significant). 
For $\Psi=15^{\circ}$, both pendulums also oscillate closely to the harmonic mode, but the oscillations contain at least two essential frequencies. In the case of $\Psi=30^{\circ}$, the oscillation picture becomes non-harmonic. This effect helps us to derive a description answering, on the engineering level, the question formulated above. Using the numeralytical approach, we can show the influence of the value $\Psi$ on the frequencies of the pendulums' oscillations. Figures 4.1-4.3 present approximated fourth-order curves for the "number of frequency peaks" versus the angle $\Psi$ for the first and second pendulums (see (4.1)). In this case, $m_{1}=m_{2}=1.57 \mathrm{~kg}, L_{1}=L_{2}=0.3 \mathrm{~m}$, and values of the angle $\Psi$ are changed in steps of $5^{\circ}$. The peaks that were included in the consideration were those covering about $95 \%$ of the energy spectrum (95\% of the area under the distribution curves of the oscillation spectrum) shown in Figures 3.1-3.5 for the two pendulums. In addition, coefficients of peak distribution curves for the pendulums (PDCP) are shown (as given in (4.1)) in Figures 4.1-4.3:

$$
\mathrm{PDCP}_{1,2}=A \Psi^{4}+B \Psi^{3}+C \Psi^{2}+D \Psi+E .
$$

Various initial conditions and parameter values for the SDP were considered in the search for analytical answers to the question formulated at the outset of the study: What is the influence of the angle $\Psi$ on the free oscillations of the mathematical model of this system? Using the number of frequency peaks comprising the frequency spectra of the pendulum oscillations as a criterion, we can provide an estimation of the behavior of the system.

In Figure 4.1, the behavior of the pendulums for which $L_{1}=L_{2}$ is shown for the cases in which $\varphi_{1} \ll \varphi_{2}, \varphi_{1}=\varphi_{2}$ and $\varphi_{1} \gg \varphi_{2}$. These cases illustrate the dependence of the shape and frequency of the pendulums' oscillations on the angle $\Psi$. In other words, the character of the oscillations changes from close to linear to strictly non-linear as the angle $\Psi$ is changing. When $L_{1} \gg L_{2}$ (Figure 4.2), the oscillations of the first pendulum are not affected by the movement of the second pendulum. However, when the initial deviation is changed from $\varphi_{1} \ll \varphi_{2}$ through $\varphi_{1}=\varphi_{2}$ to $\varphi_{1} \gg \varphi_{2}$, pendulums 1 and 2 switch roles, as is shown in Figure 4.2. From PDCP graphics, we can see the equalization trend of the distributions of the peak numbers for both pendulums for all initial conditions when $L_{2} \gg L_{1}$ (Figure 4.3).

Let us now examine the dependence of the behavior of SDPs having different parameters on the angle $\Psi$ for the linear model (small initial conditions), having two frequency peaks for each pendulum (Figures 4.4-4.6). The five curves in each graph illustrate the behavior of the pendulums depending upon different proportions between values of the pendulums' point masses. These data are correct for all small initial conditions for the SDP system. In addition, Figure 4.7 shows normalized data of comparison between the values of the point masses for the first (Figure 4.7(a)) and the second (Figure 4.7(b)) pendulums, respectively.

As we can see, the lack of analytical means for finding the shape of the function $\varphi_{i}$ $\left(t, \psi, \varphi_{10}, \varphi_{20}\right)$ (where $i$ is the number of the pendulum, and $\varphi_{10}, \varphi_{20}$ are initial deviations of the pendulums) forces the practitioner to apply a "round about way" for seeking the solution. In our case - that in which processes that are predictable in theory but for some parameters become "unpredictable" in practice - this "way" was found by applying various computations to find the dependence of the frequency content of the oscillation process on the angle $\Psi$. 


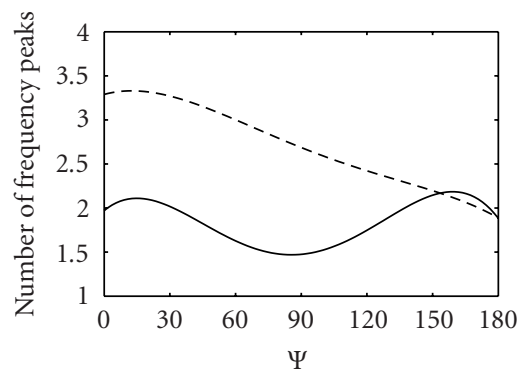

\begin{tabular}{ccc}
\multicolumn{3}{c}{$m_{1}=m_{2} ; L_{1}=L_{2} ; \varphi_{1}=\varphi_{2}$} \\
\hline Coefficients & Pendulum 1 & Pendulum 2 \\
\hline$A$ & $-3 \cdot 10^{-8}$ & $-6 \cdot 10^{-9}$ \\
$B$ & $9 \cdot 10^{-6}$ & $2 \cdot 10^{-6}$ \\
$C$ & $-9 \cdot 10^{-4}$ & $-3 \cdot 10^{-4}$ \\
$D$ & $2.04 \cdot 10^{-2}$ & $6.7 \cdot 10^{-3}$ \\
$E$ & 1.9716 & 3.2899
\end{tabular}

(a)

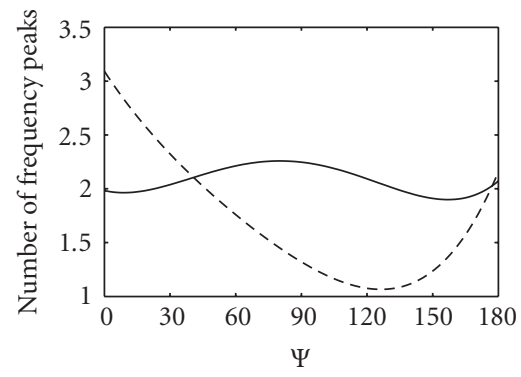

\begin{tabular}{ccc}
\multicolumn{3}{c}{$m_{1}=m_{2} ; L_{1}=L_{2} ; \varphi_{1}<\varphi_{2}$} \\
\hline Coefficients & Pendulum 1 & Pendulum 2 \\
\hline$A$ & $1 \cdot 10^{-8}$ & $7 \cdot 10^{-9}$ \\
$B$ & $-4 \cdot 10^{-6}$ & $-2 \cdot 10^{-6}$ \\
$C$ & $3 \cdot 10^{-4}$ & $-3 \cdot 10^{-4}$ \\
$D$ & $-48 \cdot 10^{-3}$ & $-31 \cdot 10^{-2}$ \\
$E$ & 1.9832 & 3.0915 \\
\hline
\end{tabular}

(b)

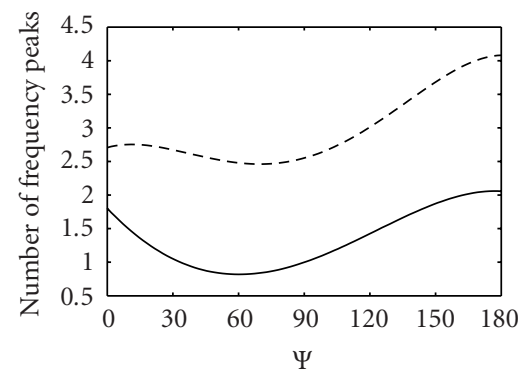

\begin{tabular}{ccc}
\multicolumn{3}{c}{$m_{1}=m_{2} ; L_{1}=L_{2} ; \varphi_{1}>\varphi_{2}$} \\
\hline Coefficients & Pendulum 1 & Pendulum 2 \\
\hline$A$ & $-3 \cdot 10^{-9}$ & $-2 \cdot 10^{-8}$ \\
$B$ & $-1 \cdot 10^{-7}$ & $5 \cdot 10^{-6}$ \\
$C$ & $3 \cdot 10^{-4}$ & $-5 \cdot 10^{-4}$ \\
$D$ & $-3.44 \cdot 10^{-2}$ & $8.8 \cdot 10^{-3}$ \\
$E$ & 1.8053 & 2.7069 \\
\hline
\end{tabular}

(c)

Figure 4.1. Peak distribution curves for pendulums (PDCP) for the cases in which $m_{1}=m_{2}$ where $0.5 \mathrm{~kg}<m_{1}<4 \mathrm{~kg}$ and $L_{1}=L_{2}$ where $0.1 \mathrm{~m}<L_{1}<5 \mathrm{~m}$ for: (a) $\varphi_{1}=\varphi_{2}$ where $5^{\circ}<\varphi_{1}<40^{\circ}$; (b) $2 \varphi_{1}<$ $\varphi_{2}<6 \varphi_{1}$ where $2^{\circ}<\varphi_{1}<15^{\circ}$; and (c) $2 \varphi_{2}<\varphi_{1}<6 \varphi_{2}$ where $2^{\circ}<\varphi_{2}<15^{\circ}$. Solid curve is PDCP of pendulum 1, and doted curve is PDCP of pendulum 2.

\section{Friction}

There are no free oscillations of the experimental device because friction always exists in practice. The value of the friction constant was obtained experimentally from the laboratory device (Figure 1.1(b)). The results obtained experimentally showed 


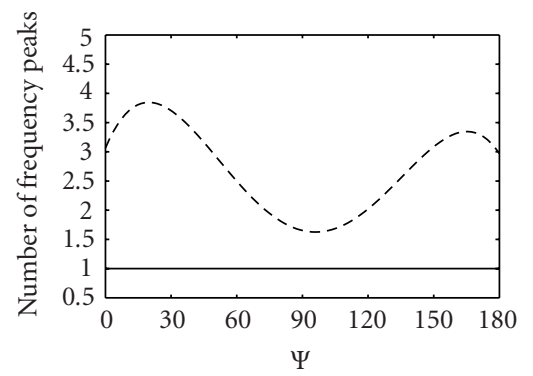

\begin{tabular}{ccc}
\multicolumn{3}{c}{$m_{1}=m_{2} ; L_{1}>L_{2} ; \varphi_{1}=\varphi_{2}$} \\
\hline Coefficients & Pendulum 1 & Pendulum 2 \\
\hline$A$ & 0 & $-7 \cdot 10^{-8}$ \\
$B$ & 0 & $3 \cdot 10^{-5}$ \\
$C$ & 0 & $-3 \cdot 10^{-3}$ \\
$D$ & 0 & $8.81 \cdot 10^{-2}$ \\
$E$ & 1 & 3.0649 \\
\hline
\end{tabular}

(a)

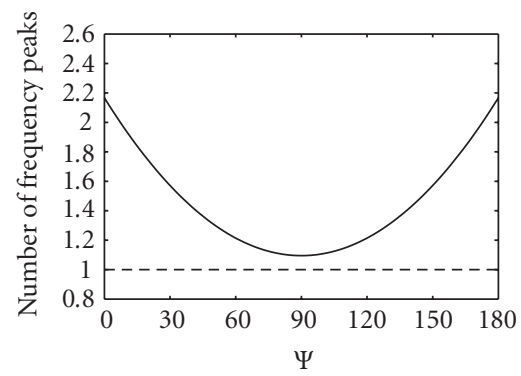

\begin{tabular}{ccc}
\multicolumn{3}{c}{$m_{1}=m_{2} ; L_{1}>L_{2} ; \varphi_{1}<\varphi_{2}$} \\
\hline Coefficients & Pendulum 1 & Pendulum 2 \\
\hline$A$ & 0 & 0 \\
$B$ & 0 & 0 \\
$C$ & $1 \cdot 10^{-4}$ & 0 \\
$D$ & $-2.38 \cdot 10^{-2}$ & 0 \\
$E$ & 2.1667 & 1 \\
\hline
\end{tabular}

(b)

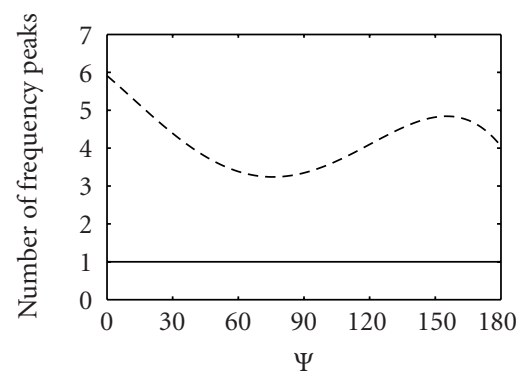

\begin{tabular}{ccc}
\multicolumn{3}{c}{$m_{1}=m_{2} ; L_{1}>L_{2} ; \varphi_{1}>\varphi_{2}$} \\
\hline Coefficients & Pendulum 1 & Pendulum 2 \\
\hline$A$ & 0 & $-3 \cdot 10^{-8}$ \\
$B$ & 0 & $9 \cdot 10^{-6}$ \\
$C$ & 0 & $-3 \cdot 10^{-4}$ \\
$D$ & 0 & $-4.9 \cdot 10^{-2}$ \\
$E$ & 1 & 1 \\
\hline
\end{tabular}

(c)

Figure 4.2. Peak distribution curves for pendulums (PDCP) for the cases in which $m_{1}=m_{2}$, where $0.5 \mathrm{~kg}<m_{1}<4 \mathrm{~kg}$ and $5 L_{2}<L_{1}<15 L_{2}$ where $0.1 \mathrm{~m}<L_{2}<5 \mathrm{~m}$ for: (a) $\varphi_{1}=\varphi_{2}$ where $5^{\circ}<\varphi_{1}<40^{\circ}$; (b) $2 \varphi_{1}<\varphi_{2}<6 \varphi_{1}$ where $2^{\circ}<\varphi_{1}<15^{\circ}$; and (c) $2 \varphi_{2}<\varphi_{1}<6 \varphi_{2}$ where $2^{\circ}<\varphi_{2}<15^{\circ}$. Solid curve is PDCP of pendulum 1 , and doted curve is PDCP of pendulum 2 .

logarithmic fading of the oscillations. If we suppose that the resistance force $F_{R}$ due to friction is proportional to the speed of motion of the pendulums, that is, $F_{R}=c \dot{\varphi}$, then the energy dissipation coefficient $\psi$ may be defined for one oscillation period $T$ as

$$
\psi=1-\left(\varphi_{1} / \varphi_{2}\right)^{2}=1-\left[\left(e^{-2 n(1+T)}\right) /\left(e^{-2 n T}\right)\right]=1-e^{-2 n T}=1-e^{-2 \lambda} .
$$




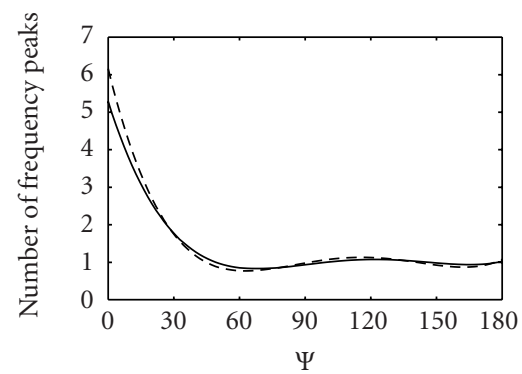

\begin{tabular}{ccc}
\multicolumn{3}{c}{$m_{1}=m_{2} ; L_{1}<L_{2} ; \varphi_{1}=\varphi_{2}$} \\
\hline Coefficients & Pendulum 1 & Pendulum 2 \\
\hline$A$ & $3 \cdot 10^{-8}$ & $5 \cdot 10^{-8}$ \\
$B$ & $-2 \cdot 10^{-5}$ & $-2 \cdot 10^{-5}$ \\
$C$ & $2.7 \cdot 10^{-3}$ & $3.6 \cdot 10^{-3}$ \\
$D$ & -0.1834 & -0.2371 \\
$E$ & 5.288 & 6.16 \\
\hline
\end{tabular}

(a)

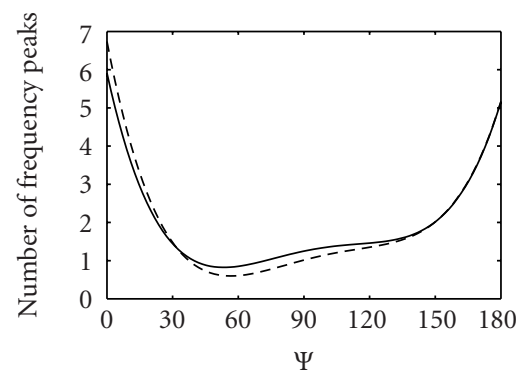

\begin{tabular}{ccc}
\multicolumn{3}{c}{$m_{1}=m_{2} ; L_{1}<L_{2} ; \varphi_{1}<\varphi_{2}$} \\
\hline Coefficients & Pendulum 1 & Pendulum 2 \\
\hline$A$ & $9 \cdot 10^{-8}$ & $9 \cdot 10^{-8}$ \\
$B$ & $-3 \cdot 10^{-5}$ & $-4 \cdot 10^{-5}$ \\
$C$ & $4.6 \cdot 10^{-3}$ & $5.1 \cdot 10^{-3}$ \\
$D$ & -0.2597 & -0.2978 \\
$E$ & 5.9114 & 6.7382 \\
\hline
\end{tabular}

(b)

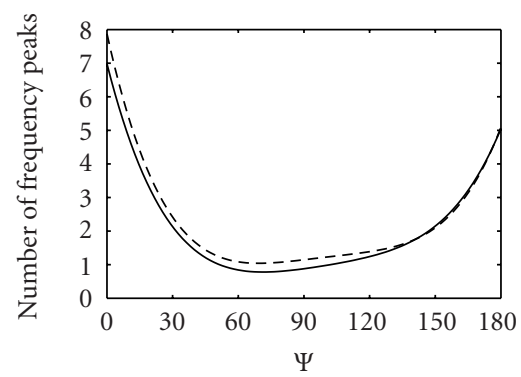

\begin{tabular}{ccc}
\multicolumn{3}{c}{$m_{1}=m_{2} ; L_{1}<L_{2} ; \varphi_{1}>\varphi_{2}$} \\
\hline Coefficients & Pendulum 1 & Pendulum 2 \\
\hline$A$ & $6 \cdot 10^{-8}$ & $8 \cdot 10^{-8}$ \\
$B$ & $-3 \cdot 10^{-5}$ & $-3 \cdot 10^{-5}$ \\
$C$ & $3.8 \cdot 10^{-3}$ & $5.1 \cdot 10^{-3}$ \\
$D$ & -0.2558 & -0.2934 \\
$E$ & 6.9806 & 7.8736 \\
\hline
\end{tabular}

(c)

Figure 4.3. PDCP graphics for the cases in which $m_{1}=m_{2}$ where $0.5 \mathrm{~kg}<m_{1}<4 \mathrm{~kg}$ and $5 L_{1}<L_{2}<$ $15 L_{1}$ where $0.1 \mathrm{~m}<L_{1}<5 \mathrm{~m}$ for: (a) $\varphi_{1}=\varphi_{2}$ where $5^{\circ}<\varphi_{1}<40^{\circ}$; (b) $2 \varphi_{1}<\varphi_{2}<6 \varphi_{1}$ where $2^{\circ}<\varphi_{1}<$ $15^{\circ}$; and (c) $2 \varphi_{2}<\varphi_{1}<6 \varphi_{2}$ where $2^{\circ}<\varphi_{2}<15^{\circ}$. Solid curve is PDCP of pendulum 1 , and doted curve is PDCP of pendulum 2.

From here, we obtain

$$
\lambda=n T=\ln \left(\varphi_{1} / \varphi_{2}\right),
$$




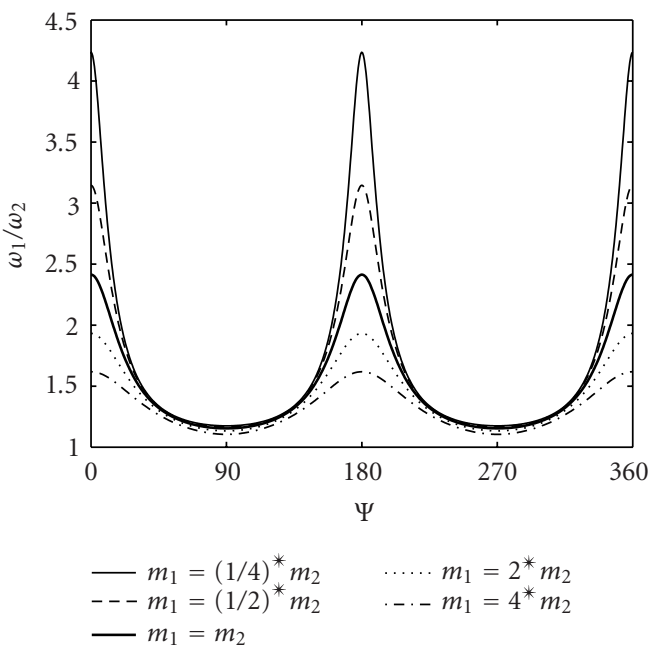

(a)

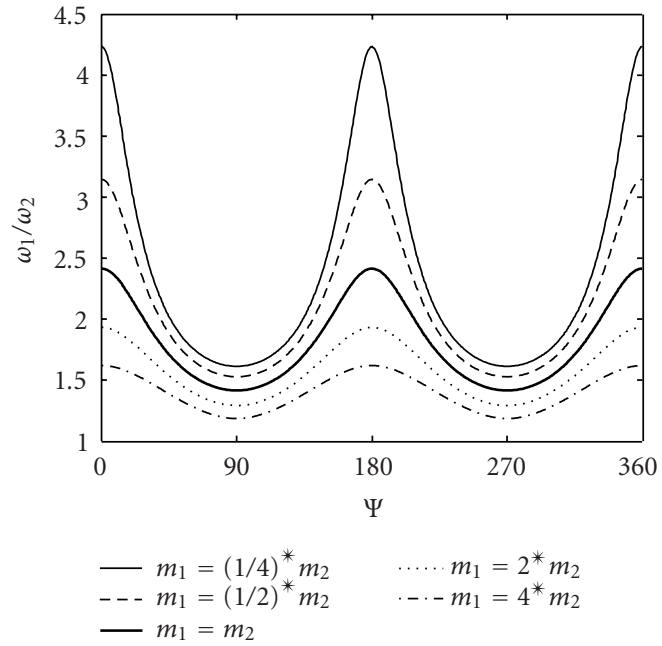

(b)

Figure 4.4. Ratio of frequencies versus angle between the oscillation planes for point masses ratio and equal length of pendulums. The initial parameters for (a) $L_{1}=L_{2}=0.2 \mathrm{~m}, m_{2}=2 \mathrm{~kg}$; and for (b) $L_{1}=L_{2}=1 \mathrm{~m}, m_{2}=2 \mathrm{~kg}$.

where $\lambda$ is the logarithmic decrement of the oscillations and $n$ is derived as

$$
2 n=\frac{c}{m L^{2}} .
$$




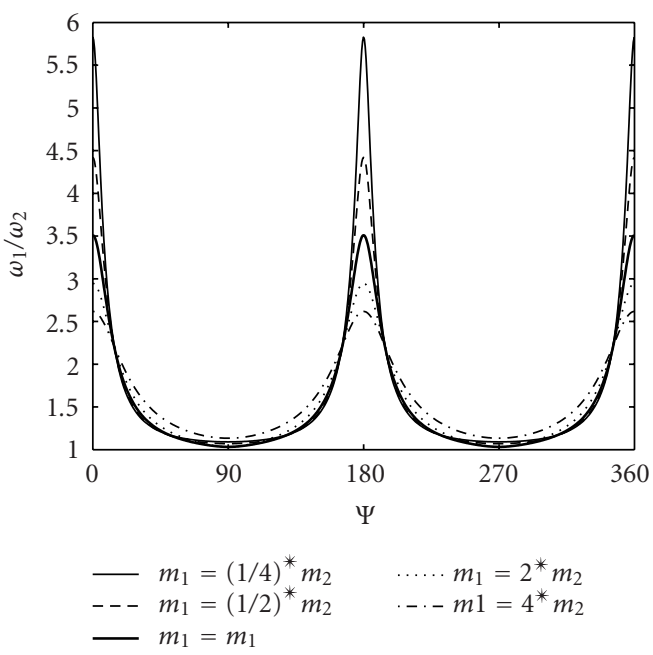

(a)

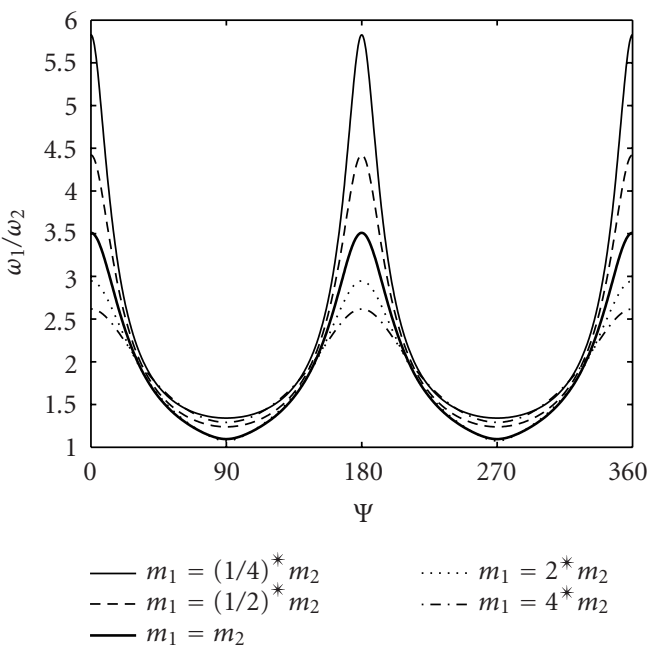

(b)

Figure 4.5. The ratio of frequencies versus angle between the oscillation planes for point masses ratio and small length of first pendulum. The initial parameters for (a) $L_{1}=0.2 \mathrm{~m}, L_{2}=5 \cdot L_{1}, m_{2}=2 \mathrm{~kg}$; and for (b) $L_{1}=1 \mathrm{~m}, L_{2}=5 \cdot L_{1}, m_{2}=2 \mathrm{~kg}$.

We may assume equal values for the friction coefficient $c$ for the hinges of both pendulums in the SDP system, but the values for the amplitudes and cycle times for the two pendulums will not be the same. Using the experimental measurements of pendulums' swinging processes and expressions (5.1)-(5.3), we determined the proportional friction coefficient of the system as $c=0.02 \mathrm{kgm}^{2} / \mathrm{s}$. The oscillations of the SDP measured 


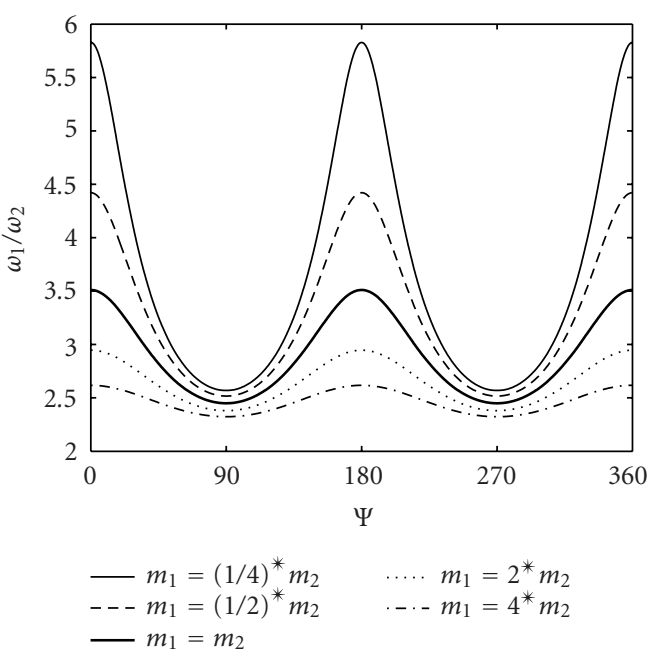

(a)

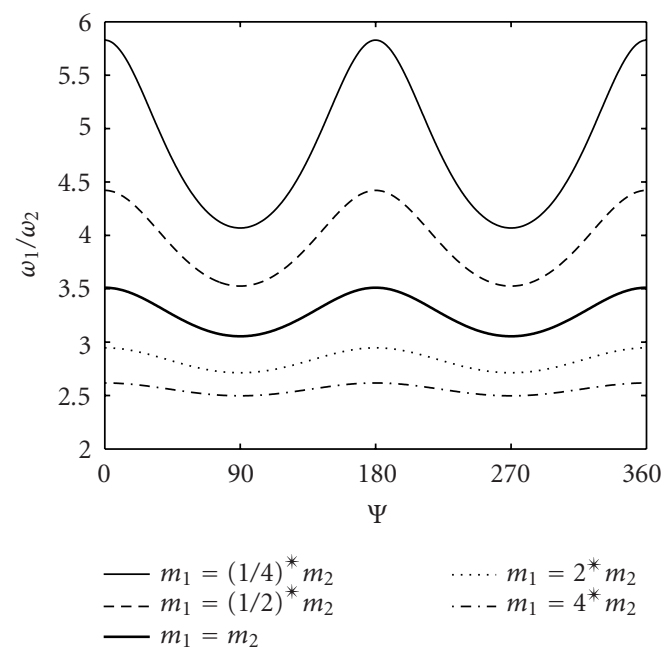

(b)

Figure 4.6. The ratio of frequencies versus angle between the oscillation planes for point masses ratio and small length of second pendulum. The initial parameters for (a) $L_{2}=0.2 \mathrm{~m}, L_{1}=5 \cdot L_{2}, m_{2}=2 \mathrm{~kg}$; and for (b) $L_{2}=1 \mathrm{~m}, L_{1}=5 \cdot L_{2}, m_{2}=2 \mathrm{~kg}$.

experimentally are presented in Figure 5.1(a), the free oscillations as calculated, in Figure 5.1(b), and the oscillations calculated taking into account the above-mentioned value of the friction coefficient, in Figure 5.1(c). We must assume that for the same initial conditions and parameters, the oscillations of pendulums are influenced only by friction of experimental system, and for other SDP set-ups these friction coefficients should be estimated experimentally. 


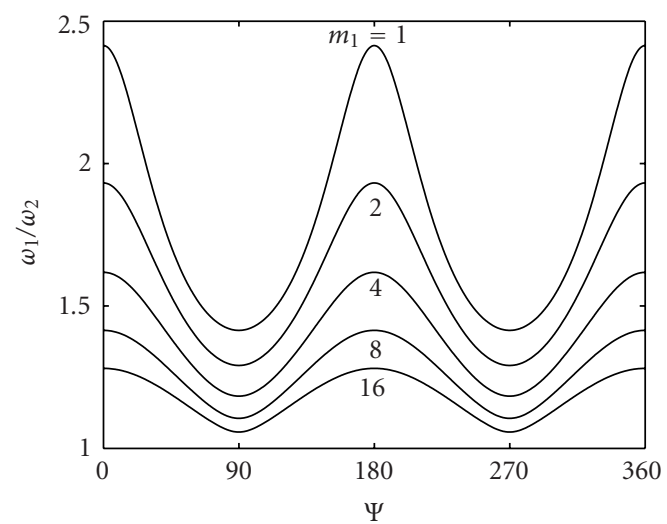

(a)

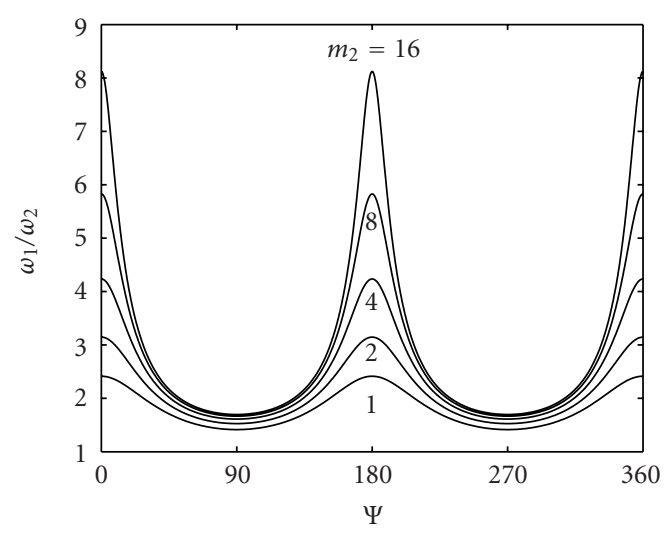

(b)

Figure 4.7. Comparison between values of the point mass for the first (a) and second (b) pendulums of SDP. The normalized initial parameters are shown on the graphs.

\section{Conclusions}

(1) We have shown that the numeralytical approach may indeed replace time- and labor-consuming process of formal calculations and enables us to obtain satisfactory engineering results for solving the very complicated problem of the behaviour of the SDP. In some cases, like those discussed here, the novelty of the computation technique lies in its ability to answer design questions, when strict mathematics cannot obviously not supply engineering solutions.

(2) The numeralytic approach may indeed be an effective tool in engineering design in, for example, the following cases:

- when the designer seeks a relatively fast, although approximate, result;

- in the analysis of nonlinear systems with many degrees of freedom. 
Approximation of the

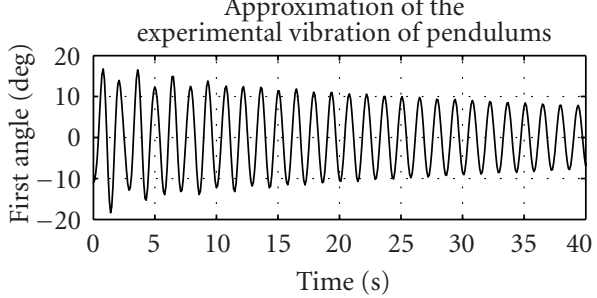

Approximation of the

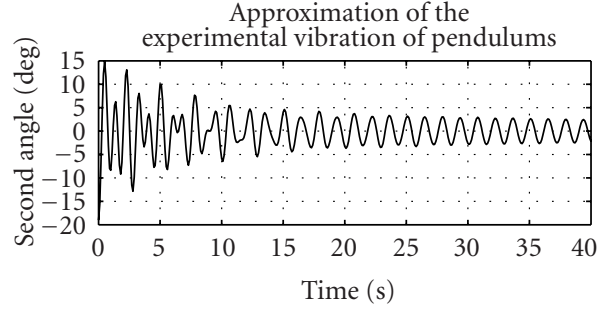

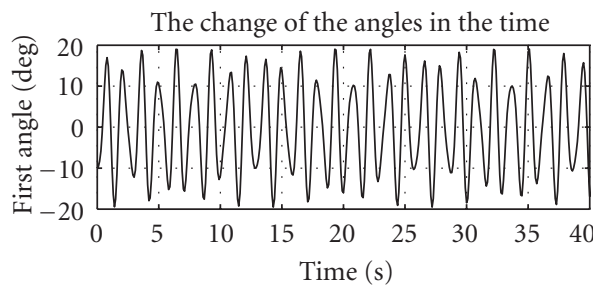

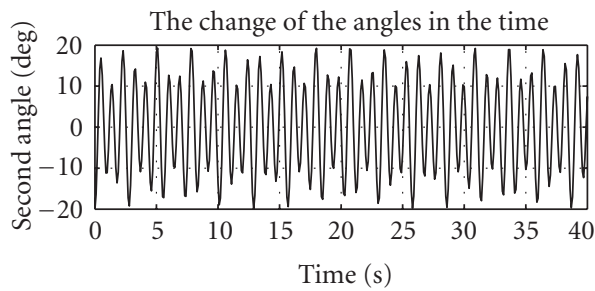

(a)

(b)
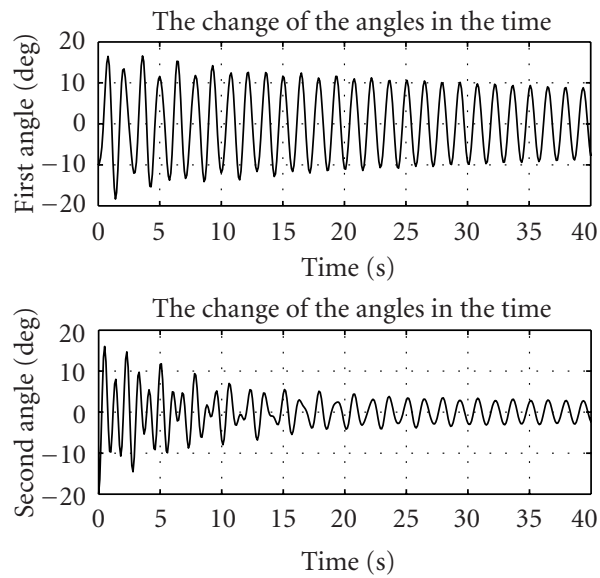

(c)

Figure 5.1. Experimental picture of the vibration of the pendulums: (a) oscillation of the experimental pendulums; (b) computed free oscillation of the pendulums; (c) computed free oscillation of the pendulums with friction estimated from experiments. The initial conditions are $\varphi_{1}=-10^{\circ}$, $\varphi_{2}=-20^{\circ}$, and initial parameters are $m_{1}=m_{2}=1.57 \mathrm{~kg}, L_{1}=L_{2}=0.3 \mathrm{~m}, \Psi=45^{\circ}, \varphi_{1}=-10^{\circ}$, $\varphi_{2}=-20^{\circ}$.

(3) Behavior of the SDP is dependent on the angle between the oscillation planes of the two pendulums whose oscillations change from almost harmonic to almost chaotic.

(4) The information gathered in the process of the SDP investigation may be applied in the design of dynamic systems, for example, robots, turbine blades, which like the SDP include bodies oscillating in different planes. 


\section{References}

[1] U. O. Akpan and M. R. Kujath, Stochastic vibration of a mobile manipulator, Transactions of the ASME. Journal of Applied Mechanics 64 (1997), no. 3, 670-675.

[2] R. Carretero-Gonzalez, H. N. Nunez-Yepez, and A. L. Salas-Brito, Regular and chaotic behaviour in an extensible pendulum, European Journal of Physics 15 (1994), no. 3, 139-148.

[3] M. G. Henders and A. C. Soudack, Dynamics and stability state-space of a controlled inverted pendulum, International Journal of Non-Linear Mechanics 31 (1996), no. 2, 215-227.

[4] M. S. Howe, The mean square stability of an inverted pendulum subject to random parametric excitation, Journal of Sound and Vibration 32 (1974), 407-421.

[5] E. R. Lowenstern, The stabilizing effect of imposed oscillations of high frequency on a dynamical system, Philosophical Magazine 13 (1932), 458-486.

[6] P. Lynch, Resonant motions of the three-dimensional elastic pendulum, International Journal of Non-Linear Mechanics 37 (2002), no. 2, 345-367.

[7] R. Mitchell, Stability of the inverted pendulum subjected to almost periodic and stoichastic base motion - an application of the method of averaging, Journal of Sound and Vibration 32 (1974), $101-123$.

[8] A. H. Nayfeh, Introduction to Perturbation Techniques, John Wiley \& Sons, New York, 1981.

[9] R. E. O’Malley Jr., Introduction to Singular Perturbations, Applied Mathematics and Mechanics, vol. 14, Academic Press, New York, 1974.

[10] F. M. Phelps III and J. H. Hunter Jr., An analytical solution of the inverted pendulum, American Journal of Physics 33 (1965), 285-295.

[11] M. G. Rusbridge, Motion of the sprung pendulum, American Journal of Physics 48 (1980), no. 2, $146-151$.

[12] B. Z. Sandler, Numeralytics: A computation approach for engineering practitioners, ASME Journal of Mechanical Design 124 (2002), no. 2, 151-157.

[13] B. A. Schmidt, The rotationally flexible pendulum subjected to a high frequency excitation, Transactions of the ASME. Journal of Applied Mechanics 57 (1990), no. 3, 725-730.

[14] __ Analysis of a pendulum on a rotating arm, Transactions of the ASME. Journal of Applied Mechanics 59 (1992), 233-234.

[15] A. Stephenson, On a new type of dynamical stability, Memoirs and Proceedings of the Manchester Literary and Philosophical Society 52 (1908), no. 8, 1-10.

S. Bendersky: Pearlstone Center for Aeronautical Engineering Studies, Department of Mechanical Engineering, Ben-Gurion University of the Negev, P.O. Box 653, Beer-Sheva 84105, Israel E-mail address: bendersk@bgumail.bgu.ac.il

B. Sandler: Pearlstone Center for Aeronautical Engineering Studies, Department of Mechanical Engineering, Ben-Gurion University of the Negev, P.O. Box 653, Beer-Sheva 84105, Israel E-mail address: sandler@bgumail.bgu.ac.il 


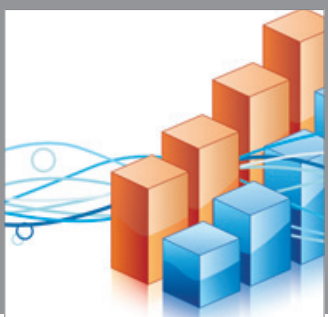

Advances in

Operations Research

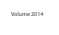

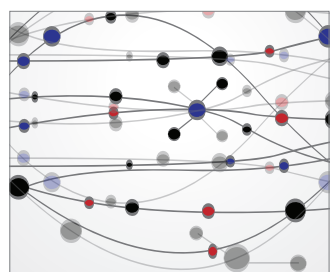

\section{The Scientific} World Journal
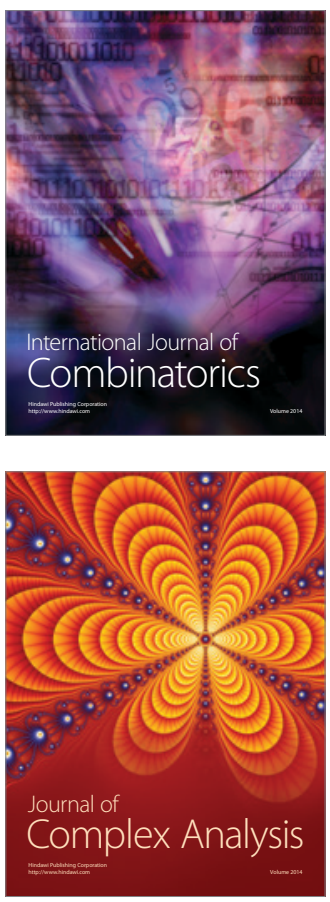

International Journal of

Mathematics and

Mathematical

Sciences
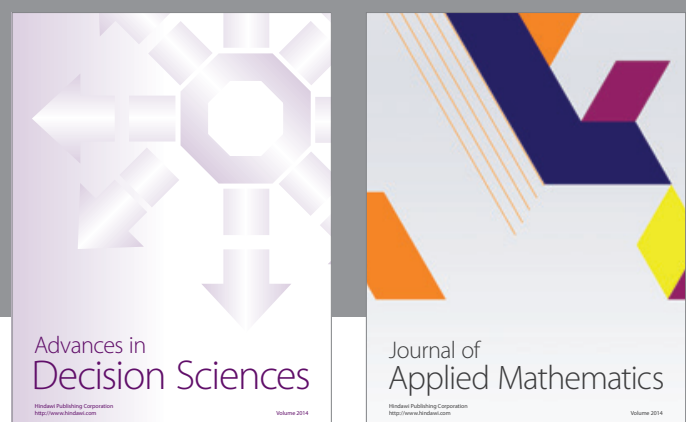

Journal of

Applied Mathematics
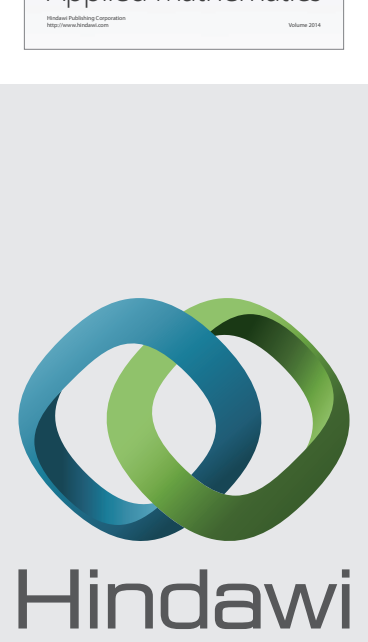

Submit your manuscripts at http://www.hindawi.com
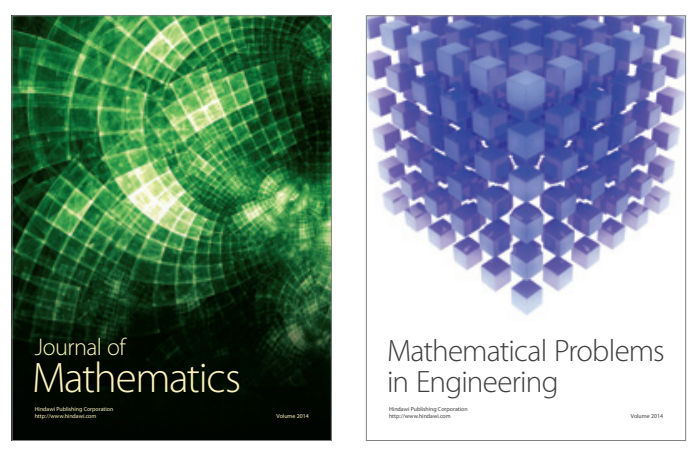

Mathematical Problems in Engineering
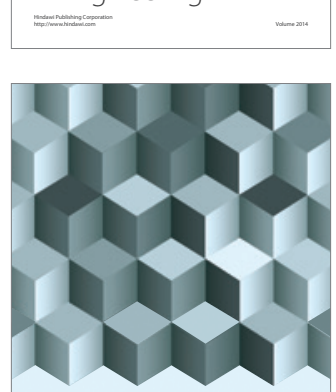

Journal of

Function Spaces
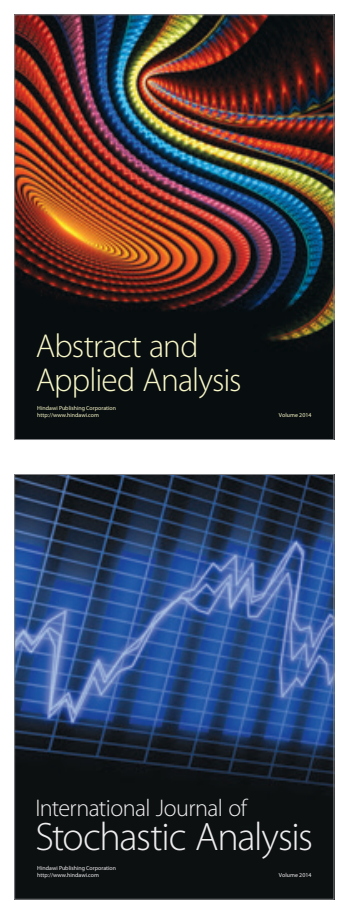

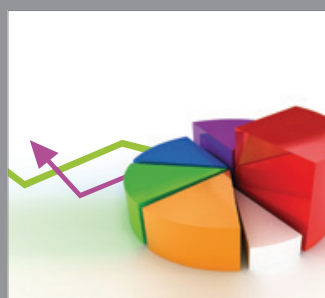

ournal of

Probability and Statistics

Promensencen
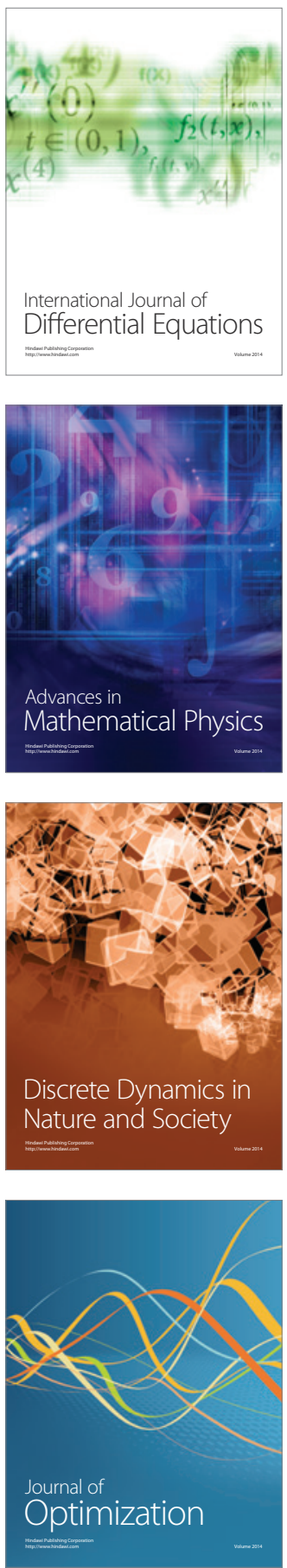